\section{Integrative Medicine \\ International}

\title{
The Puzzle of the Skin Patterns
}

\author{
Daniela Tenea \\ Department of Dermatology, Steve Biko Academic Hospital, University of Pretoria, Pretoria, \\ Republic of South Africa
}

\section{Keywords}

Skin mosaicism · Blaschko's lines · Immunocompromised district · Isotopic response ·

Koebner phenomenon

\section{Abstract}

Background: The pattern of distribution of skin diseases remains one of the most elusive problems in dermatology, particularly when multiple pathologies are involved and coexistence of patterns is present. Most skin diseases show a predilection for certain body areas and are located in characteristic patterns. Many dermatoses exhibit lesions in a segmental fashion concomitantly with disseminated patterns. Sometimes the sites of predilection are inexplicable. Objectives: The main purpose of this paper is to give an overview on the peculiar arrangement of cutaneous lesions in both congenital and acquired skin disorders as well as reactive phenomena in vulnerable areas, in order to better recognise them clinically and understand their pathogenesis. Methods: Based on personal observations and a review of literature, this paper summarises the basics in understanding the patterned distribution of skin lesions, highlights the significance of the lines of Blaschko and their relationship with mosaicism, and describes some of the reactive skin phenomena. Results: Skin mosaicism plays a crucial role in the distribution and arrangement of skin lesions, as demonstrated by recent advances in genetic research in an increasing number of various skin diseases.

(C) 2016 The Author(s)

Published by S. Karger AG, Basel

\section{Introduction}

The distribution pattern of skin diseases remains an unexplained oddity or pathogenetically misunderstood. Pattern analysis of skin lesions is of great assistance in clinical diagnosis for every dermatologist (recognition of both primary and secondary lesions, description of individual skin lesion types, size, shape, colour, texture changes, surface characteristics, and the presence or absence of pain or itch). 
Most skin diseases are not randomly distributed over the body. Some show diagnostic sites of predilection (pityriasis rosea, scabies, dermatitis herpetiformis). For others, history reveals possible triggering factors (UV light exposure, trauma, cold exposure, drug intake, travelling) determining certain distributions of the lesions. Regional body temperature variations favouring particular skin lesion localisation have attracted the attention of some investigators trying to explain the distribution of lesions in leprosy or gout tophi in cold areas. Other skin diseases are even more enigmatic in their distribution characteristics because their lesions follow specific virtual lines covering the human body [1, 2].

Three major line patterns cover the human body: the dermatomes (Head zones), the relaxed skin tension lines (Langer's lines), and the naevoid lines of Blaschko, indicating a neural, haematogenic, or embryogenic background in their pathogenesis $[1,2]$.

\section{Head Zones (Dermatomes)}

Head zones refer to dermoneural units supplied by one segmental sensory nerve. Louis Bolk, a professor of anatomy in Amsterdam, was one of the earliest anatomists who illustrated the metamerism and segmentation in human skin. Henry Head, an English neurologist, matched the spinal nerve territories with postmortem confirmation of inflamed or damaged posterior root ganglion in herpes zoster. Head's lines run transversally over the trunk, but follow a zig-zag or S-shaped configuration on the head and limbs (herpes zoster, notalgia paresthetica) $[2,3]$.

\section{Langer's Lines (Tension Lines)}

Langer's lines were first described in 1861 by the Austrian anatomist Karl Langer, who gave credit to the French surgeon Guillaume Dupuytren as the first to recognise the lines. The clinical relevance of the pattern of these lines is mostly surgical, as they correspond to the direction in which the vector of naturally occurring tension of the skin is minimal, promoting better wound healing [2]. Langer's lines show 3 major patterns: V-shaped on the upper chest and back, circumferential around the shoulders and hips, and transversal on the lower back and abdominal wall [2].

The Christmas tree pattern is the thoracodorsal expression of Langer's lines, best represented in pityriasis rosea, mycosis fungoides, maculopapular eruption of secondary syphilis, the early stage of guttate psoriasis, Kaposi sarcoma idiopathicum multiplex, paraneoplastic eruption of multiple seborrhoeic keratosis known as Leser-Trélat sign, and juvenile papulonodular urticaria pigmentosa [2].

\section{Lines of Blaschko}

The lines of Blaschko were first described in 1901 by the German dermatologist Alfred Blaschko, who published a book comprising hand drawings of linear lesions of the skin from various parts of the body and a unique collection of 175 patients with epidermal naevi, sebaceous naevi, or naevus angiolipomatosus, as well as acquired linear skin diseases such as linear psoriasis, band-like scleroderma, linear lichen planus, or linear eczema (Fig. 1) [2, 4]. They are known as border lines of epidermal genetic aberrations caused by genetic mosaicism occurring in the early stages of embryogenesis $[1,2,4]$.

Blaschko's lines involve the epidermis and its appendages, melanocytes, and connective tissue elements either separately or in combination, explaining their diverse clinical 
Fig. 1. Lines of Blaschko reproduced from the original drawing, completed on the head according to more recent data [4].

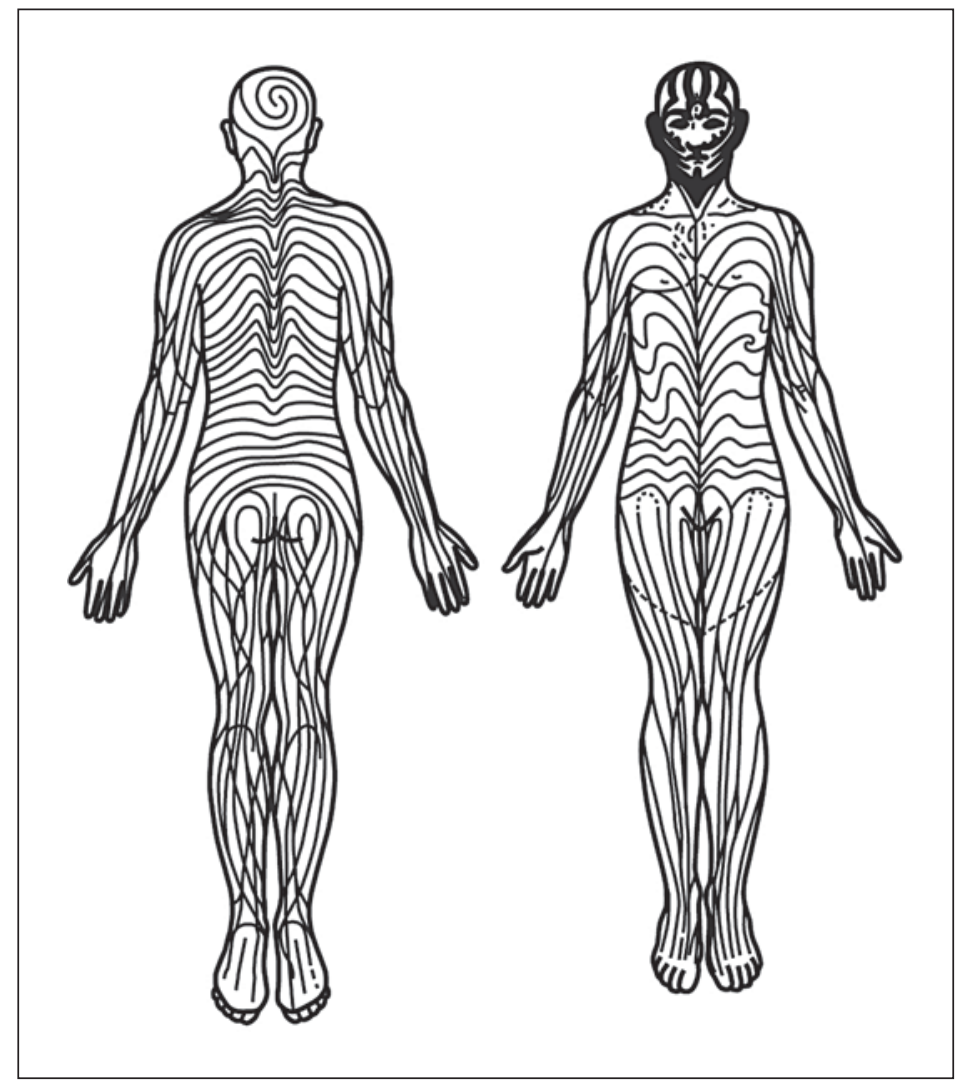

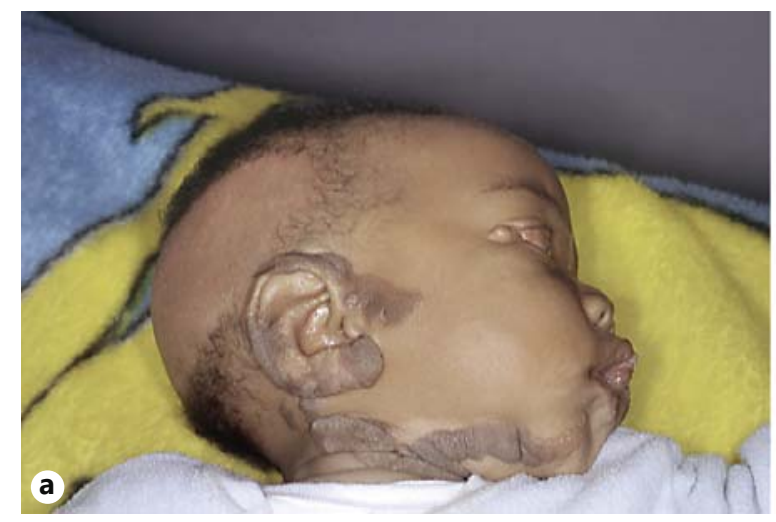

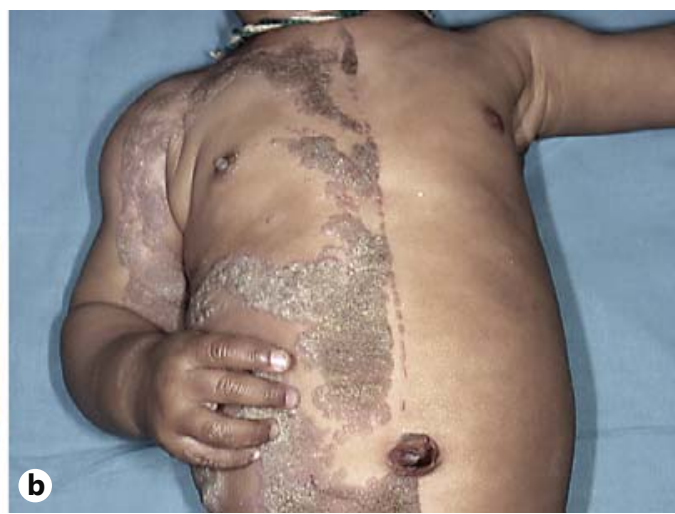

Fig. 2. Systematised epidermal naevus that demonstrates the distribution in lines of Blaschko of the face (a) and trunk (b).

expression: hyperpigmentation (linear epidermal naevus, Fig. 2), hypopigmented streaks (lichen striatus), whorls (incontinentia pigmenti, Fig. 3a), verrucous inflamed appearance, dermal atrophy (atrophodermia linearis of Moulin), papules (linear lichen planus), dyskeratosis (linear Darier disease), vascular changes (naevoid telangiectasias), and lobulated, cerebriform pattern (naevus sebaceus, Fig. 3b). Benign neoplasms following the lines of Blaschko have been added to the list: eccrine poromas, syringomas, and multiple trichoepitheliomas [2-4]. 
Fig. 3. a Hypopigmented whorls of incontinentia pigmenti achromians in an infant girl. b Naevus sebaceus affecting one side of the scalp and face. Note the characteristic lobulated pattern.

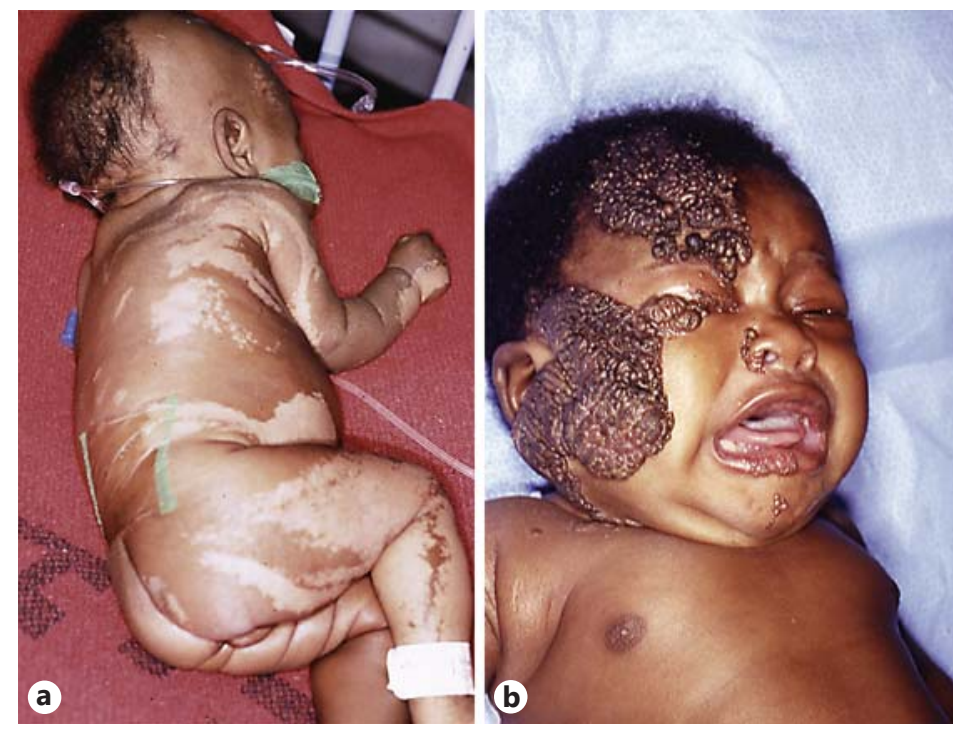

The lines reflect the route of embryonic cell migration, best shown as stripes on the extremities, whorls or S-shaped configuration on the anterior trunk, bizarre fountain-like or $\mathrm{V}$-shaped pattern on the back, and inverted U-shape pattern from the breast area onto the upper arms $[1,3,4]$. The head and neck Blaschko's lines have a more complex hour-glass configuration, converging at the nasal root and intersecting in several areas on the face. They are spiral on the scalp $[1,5]$.

The lines of Blaschko do not follow any known nervous, vascular, or lymphatic structures in the skin. Although they can be matched to the course of dermatomes, they do not correspond to them. The lines of Blaschko are to be distinguished from dermatomes, Voigt's lines (borders between areas of innervation by peripheral cutaneous nerves), Langer's lines of cleavage, embryonic clefts, and pigmentary demarcation lines. Defects arising from neural crest abnormalities should not be mistaken for Blaschko's lines. The resulting pattern may involve similar tissue structures, but with different phenotype expressivity [3, 4]. Extracutaneous analogies of Blaschko's lines have also been described in the retina (X-chromosomal oculocutaneous albinism), lens (X-chromosomal chondrodysplasia punctata and cataract), iris (X-linked oculocutaneous albinism), teeth (X-linked amelogenesis imperfecta), and bone (osteopathia striata with cranial sclerosis) [6].

Acquired inflammatory dermatoses may also feature a Blaschko linear pattern, and the generic term of adult blaschkitis or BLAISE (Blaschko linear acquired inflammatory skin eruption) has been applied for some of them. They include linear forms of atopic dermatitis (lichen striatus), linear lichen planus, naevoid psoriasis, linear vitiligo, morphea en coup de sabre, fixed drug eruption, erythema multiforme, graft-versus-host disease, and linear and whorled naevoid hypermelanosis [7].

Mixed cases showing coexistence of both segmental and non-segmental involvement usually remain unexplained. They tend to appear at a younger age or to be present at birth and often precede the appearance of non-segmental lesions; they are more pronounced and extremely difficult to treat [8]. Family members may show the disease in its non-segmental form. Such pronounced segmental involvement has been explained by the assumption that at an early developmental stage, loss of heterozygosity involving one of the predisposing genes occurred in a somatic cell and gave rise to a patch of homozygous or hemizygous tissue $[7,8]$. 
Table 1. Classification of polygenic inflammatory diseases distributed along the lines of Blaschko

Type 1: Disease involvement occurs exclusively along the lines of Blaschko (within a time frame which can never be ascertained since the patient can still develop non-segmental disease later in life)

Type 1a: A non-segmental distribution exists (lichen planus, psoriasis)
Type 1b: Blaschko linearity is exclusively manifested, and non-segmental manifestation is either not known or does not exist (lichen striatus/ adult-onset blaschkitis)

Type 2: Blaschko linear involvement of the disease and its non-segmental manifestation occur simultaneously or successively

Type 2a: Refers to the skin involvement of the same type as the one manifested in the linear distribution (psoriasis, lichen planus)
Type $2 \mathrm{~b}$ : Includes other clinical and biological manifestations of the disease occurring either on the skin or systemically (linear systemic lupus erythematosus or linear dystrophic calcifications of dermatomyositis in a patient with either clinical/ biological signs of systemic lupus erythematosus or dermatomyositis, respectively)

A genetically orientated concept of superimposed segmental manifestation of polygenic skin disease was proposed by Rudolf Happle in his attempt to elucidate the genetic basis for common acquired skin diseases, such as atopic dermatitis, psoriasis, lichen planus, vitiligo, or granuloma annulare, occurring in a linear pattern [8]. The concept was further expanded by Lipsker and Lenormand [9], who proposed a descriptive, sign-based classification of polygenic inflammatory diseases distributed along the lines of Blaschko (Table 1).

Besides these skin patterns, several dermatological phenomena have been described in the literature as responses to various insults showing peculiar skin arrangement: the isotopic response of Wolf, the isomorphic Koebner phenomenon, their inverse variants (the reverse isotopic response or non-response and Renbok phenomenon), and the photo recall phenomenon [10-15]. These are not diseases, but define a reaction pattern of a secondary skin disease developing within a variable period of time (days, months, years, decades) after the initial insult.

A regional immune dysregulation, recently labelled by Ruocco et al. [10] as immunocompromised district (ICD), may occur even in immunocompetent individuals. ICD is an umbrella term for skin areas more vulnerable to genetic and acquired insults than the rest of the body. The factors responsible for localised immune dysregulation are various and most of the time identifiable by careful clinical history: skin barrier disturbances (structural or functional), cutaneous mosaicism, herpes-infected sites, chronic lymphedema, or heterogeneous skin injuries [10-12].

Cutaneous mosaicism is the strongest genetic example of how a cutaneous site populated by cells with distinct antigenic or immunologic properties differs in its susceptibility or resistance to skin disorders $[1,11,16]$.

Cutaneous areas that have been infected by herpes viruses (mainly varicella zoster virus) are known to be privileged sites for either selectively harbouring skin diseases (malignancies, infections, immune dysregulation) not appearing elsewhere (locus minoris resistentiae, $1 \mathrm{mr}$ ) or selectively rejecting skin diseases involving the rest of the body (locus majoris resistentiae, LMR) [11-15, 17] (Fig. 4).

The Wolf isotopic response describes the occurrence of a new unrelated disease at the same location as the previously healed disease (isotopic means "at the same place"). The term was coined in 1995 by Ronni Wolf, who described several types of cutaneous lesions occurring within the areas of cleared herpes zoster (majority) or, less frequently, herpes simplex [1315]. The phenomenon does not seem to be rare, but has been overlooked or underreported. 
Fig. 4. a Wolf postherpetic isotopic response. Leukaemia cutis developed in healed herpes zosterophthalmic division of the trigeminal nerve. bWidespread purpuric macules and patches of StevensJohnson syndrome triggered by carbamazepine, sparing the right thoracic skin area previously affected by herpes zoster (reverse isotopic phenomenon) [22].
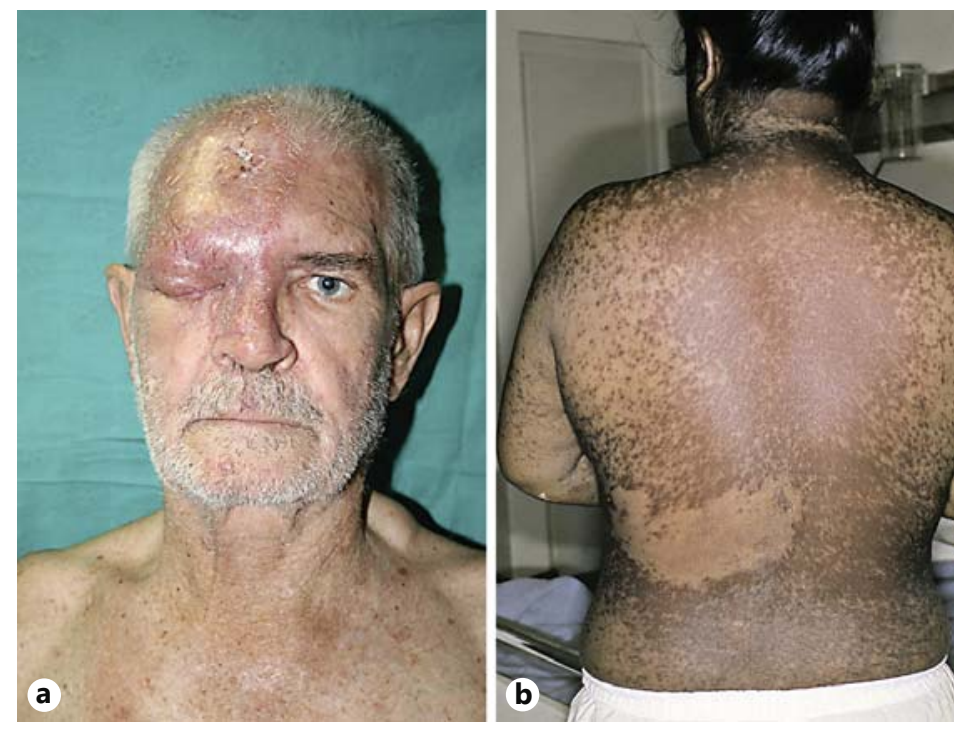

By 2014, 189 patients with Wolf postherpetic isotopic response had been reported (169 after varicella zoster virus infection and 20 after herpes simplex infection) $[14,15]$.

The postherpetic isotopic reactions reported to date include granulomatous reactions of various types (majority), malignant tumours, leukaemic or lymphomatous infiltrations, infections, dysimmune reactions, and comedonic-microcystic reactions [12, 15, 17] (Fig. 4). The majority of these disorders have been reported in immunosuppressed patients with carcinomas, lymphomas, leukaemias, HIV infection, or autoimmune dysregulations, but other patients showed no apparent underlying disease.

The pathomechanisms of Wolf postherpetic isotopic responses have not been fully elucidated (viral, neural, vascular, or immunologic aetiologies have been proposed). Locally hampered lymphatic drainage seen in chronic lymphedema as well as postoperative or posttraumatic lymph stasis may also hinder the normal trafficking of immunocompetent cells and act as cofactors in the Wolf phenomenon [12-14].

Skin areas that have been subjected to any kind of trauma (radiation dermatitis, stasis ulcers, chemical or thermal burns, vaccination site, tattoos, and amputation stumps) are fertile ground for the development of malignancy $[12,13,18,19]$ (Fig. 5).

The list of cutaneous insults may be extended to UV or ionising radiation, and new conceptual terms emerge as coined by Ruocco et al. [10] such as: (1) isophototopic response: onset of a new skin disease (infections, tumours, dysimmune reactions) in the skin areas exposed to a natural (sun) or artificial (UV lamps) source of UV radiation (lmr); (2) isophototopic non-response or localised cutaneous photoanergy: a skin area exposed to a natural (sun) or artificial (UV lamps) source of UV radiation is selectively spared by a diffuse skin disease (LMR); (3) isoradiotopic response: the onset of a new disease (infection, tumour, or immune dysregulation) on the skin area previously exposed to ionising radiation ( $\mathrm{lmr}$ ); (4) isoradiotopic non-response: a skin area exposed to ionising radiation is selectively spared by a diffuse skin disease LMR); (5) isocaumatopic response: onset of a new skin disorder on a burn scar (lmr); (6) isocaumatopic nonresponse: a burned skin area is selectively spared by a diffuse skin disorder (LMR); (7) isotraumatopic response: onset of a new skin disorder on a traumatised area ( $\mathrm{lmr}$ ); (8) isotraumatopic non-response: a traumatised skin area is selectively spared by a diffuse skin disorder (LMR).

The isomorphic response of Koebner, meaning the appearance of the same disease at another location, is perhaps the most commonly reported phenomenon. It is a classic example 

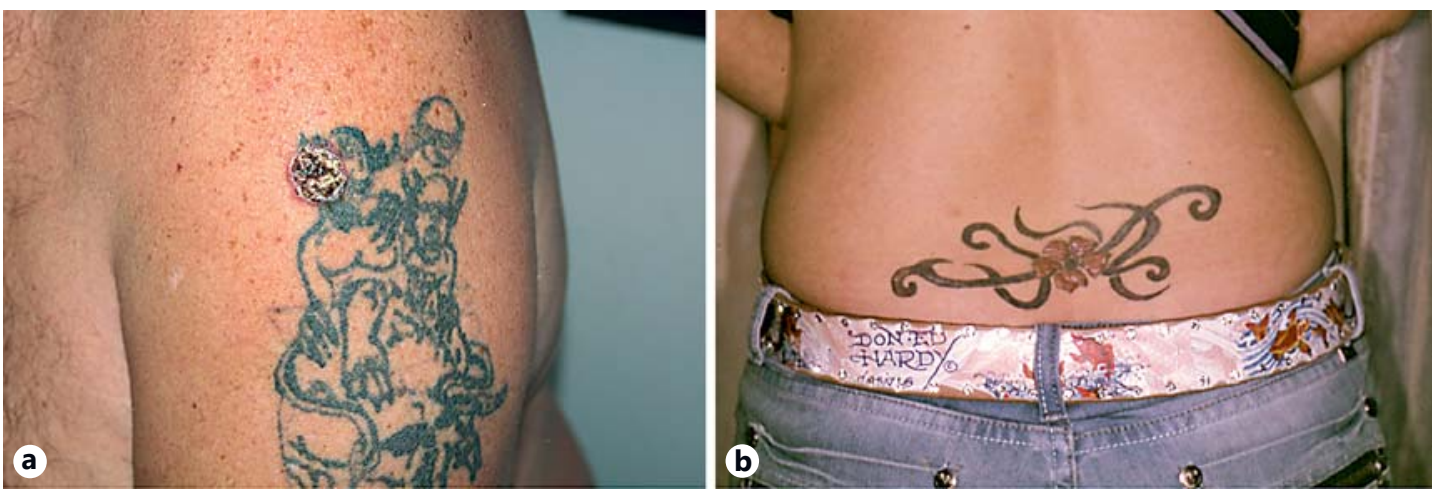

Fig. 5. a Basal cell carcinoma that developed in a tattoo area of the arm ( $\mathrm{lmr}$ ). b Mycosis fungoides developed only in the red tattoo area of the lower back, sparing the blue tattoo areas (lmr).

of lmr. Initially described in association with psoriasis, Koebner phenomenon has been reported in a variety of non-infectious inflammatory diseases such as lichen planus, vitiligo, systemic lupus erythematosus, pityriasis rosea, or dermatitis herpetiformis [20].

The triggers of Koebner phenomenon cited in the literature are various and non-specific: environmental (heat, cold, UV exposure, ionising radiation), trauma (scratches, bites, needling, burns, tattoos, nail manicuring, shaving, thumb suckling), scars (postoperative, after burns, vaccination), and therapeutics (iodine application, Roentgen therapy, withdrawal of methotrexate therapy) $[18,20]$ (Fig. 6).

The latent period between the injury to uninvolved skin and the appearance of disease is usually 14 days. It is believed that when the disease is unstable, at an early age of onset or flaring, Koebner phenomenon is more likely to occur [20].

A pseudo-isomorphic response of Koebner is considered to appear in molluscum contagiosum, warts, Behçet disease, and pyoderma gangrenosum, where the infectious organisms rather spread following trauma [20].

The mutually interactive Koebner phenomenon describes the concomitant occurrences of 2 different diseases in the same areas in which Koebner reaction has been observed: lupus erythematosus with lichen planus, psoriasis with lupus erythematosus, or sarcoidosis with lupus erythematosus [20]. Occasional localisation of the lesions to areas of trauma has been reported in Darier disease, erythema multiforme, Hailey-Hailey disease, Kyrle disease, lichen sclerosus, perforating folliculitis, and reactive perforating collagenosis [20]. Immune dysregulation in a cutaneous district, either impaired (leading to opportunistic infections and tumours) or exacerbated (bullous pemphigoid, lichen planus) accounts for strongly supported examples of reverse Koebner phenomenon or Renbok reaction. This was originally reported by Happle et al. [21] in patients having concomitant alopecia areata and psoriasis or seborrhoeic dermatitis. This reverse reaction is seen when an area of psoriasis clears following injury. Possible explanations may be that either the lesions of psoriasis or seborrhoeic dermatitis may have a protective effect with regard to alopecia areata, or conversely the hair disease prevented the development of these inflammatory skin disorders [21].

Pathogenically unrelated diseases may follow a zosteriform distribution if they are linked to a preceding herpes zoster infection by Koebner phenomenon, Renbok phenomenon, or an isotopic response [22, 23] (Fig. 4b).

Sparing phenomena, increasingly reported in the dermatological literature, direct the clinician's attention towards a conspicuous absence of lesions in an ICD [16]. 
Fig. 6. Plaques of psoriasis on pressure areas of the stump in an amputated leg (Koebner phenomenon).

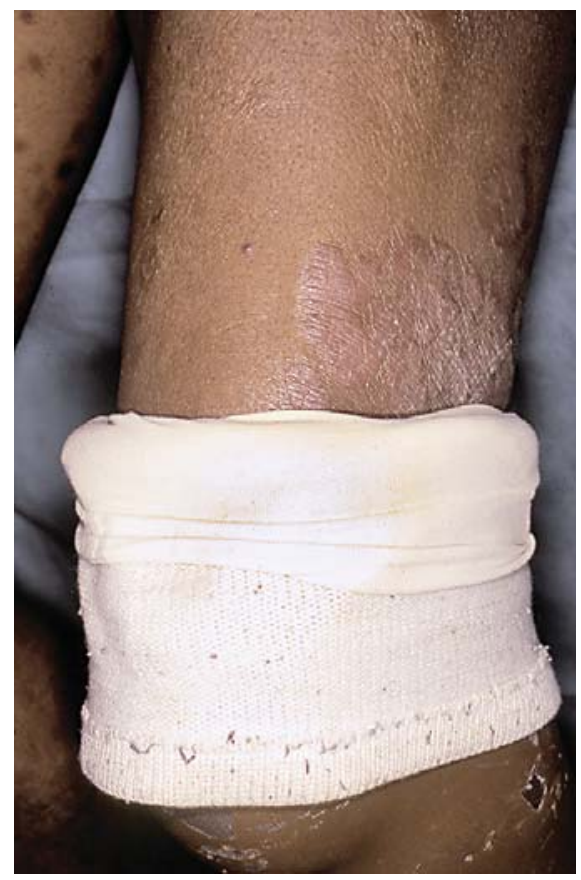

\section{Discussion}

The list of the patterned skin disorders that we attempted to describe in this review is far from being complete. They are diverse and have various degrees of severity. Trying to explain their distribution and understand their pathogenesis directs our attention towards cutaneous mosaicism as a common denominator for both monogenic and polygenic skin disorders and cutaneous reactive patterns in vulnerable areas.

Owing to assiduous genetic research for the past 2 decades, the concept of cutaneous mosaicism became a reality, being demonstrated in an increasing number of various skin diseases. Happle has mastered the concept that cutaneous lesions following the lines of Blaschko are manifestations of somatic mosaicism. He gave a new definition for naevi and stated that all naevi are mosaics, and recently proposed a new classification of different categories of skin mosaicism [24].

Mosaicism describes an organism composed of a mixture of 2 or more cell populations with distinct genotypes and concomitant patterned phenotypes (lines of Blaschko) originating from a single fertilised egg $[3,5,24,25]$. It is a biological phenomenon that results when a postzygotic mutation occurs early during embryogenesis in gonadal cells (germline mosaicism) or other cells (somatic mosaicism). The pattern of the cutaneous mosaicism depends on the type of cell that is affected and its trajectory of migration and proliferation during embryogenesis $[5,26]$.

In contrast, a genetic chimera is composed of cells derived from different zygotes. It is a rare event that occurs when there is a dual fertilisation of the egg by 2 sperms or fusion of 2 zygotes $[25,26]$. Spontaneous cell mixtures in chimeras may also cause the skin to manifest 2 shades of colour and different skin patterns, such as the flag-like rectangular pattern (generalised naevus spilus), a pattern of round patches similar to café-au-lait lesions in von Recklinghausen disease, and a striate pattern imitating the systematised epidermal naevus [26].

Five different skin patterns of mosaicism have been recognised so far $[5,24,26,27]$ (Fig. 7): (1) type 1 (Blaschko linear skin disorders) having 2 subtypes: type 1a or narrow- 

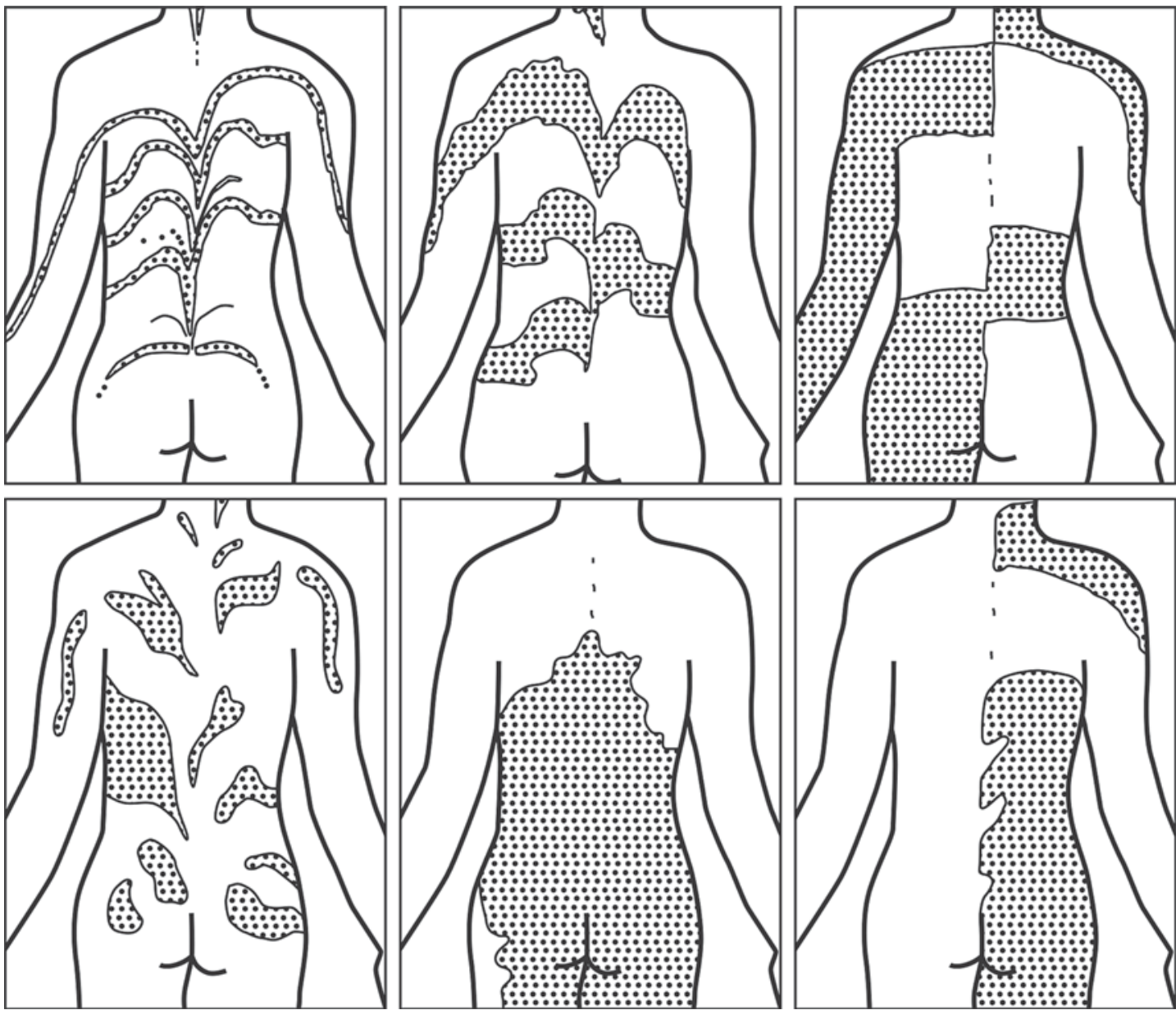

Fig. 7. Pigmentary patterns associated with human mosaicism [24]. Type 1a, lines of Blaschko, narrow-band pattern (top left). Type $1 \mathrm{~b}$, lines of Blaschko, broad-band pattern (top centre). Type 2, checkerboard pattern (top right). Type 3, phylloid pattern (bottom left). Type 4, patchy pattern without midline separation (bottom centre). Type 5 , patchy pattern with lateralisation (bottom right).

band pattern (incontinentia pigmenti), and type 1b or broad-band pattern (McCune-Albright syndrome); (2) type 2: checkerboard or flag-like pattern with sharp midline separation (speckled lentiginous naevi, Becker naevi, port-wine stains, cutis marmorata telangiectatica), the pigmentary variation in a human chimera most often displaying this pattern; (3) type 3: phylloid pattern, referring to leaf-like, pear-shaped lesions (phylloid hypomelanosis); (4) type 4: patchy pattern without midline separation (giant congenital melanocytic naevi) (Fig. 8); (5) type 5: patchy pattern with lateralisation (unique to CHILD syndrome) (Fig. 9). A detailed overview of the different forms of mosaicism is beyond the scope of this article, and the reader is referred to other sources that deal with the subject in detail $[5,24$, 26, 27].

Generally speaking, one can distinguish 2 major subtypes of mosaicism: (1) Genomic mosaicism is caused by de novo alteration in the DNA sequences (affecting genes or chromosomes), leading to a genetically heterogeneous organism. Defects that occur early during embryonic development (loss of heterozygosity, gene mutations, chromosomal abnormalities) can affect the skin in a mosaic pattern [26, 27]. (2) Functional (epigenetic) mosaicism results from changes in gene expression that are passed on from one generation to the next, 
Fig. 8. Giant congenital melanocytic naevus in an infant with Down syndrome.
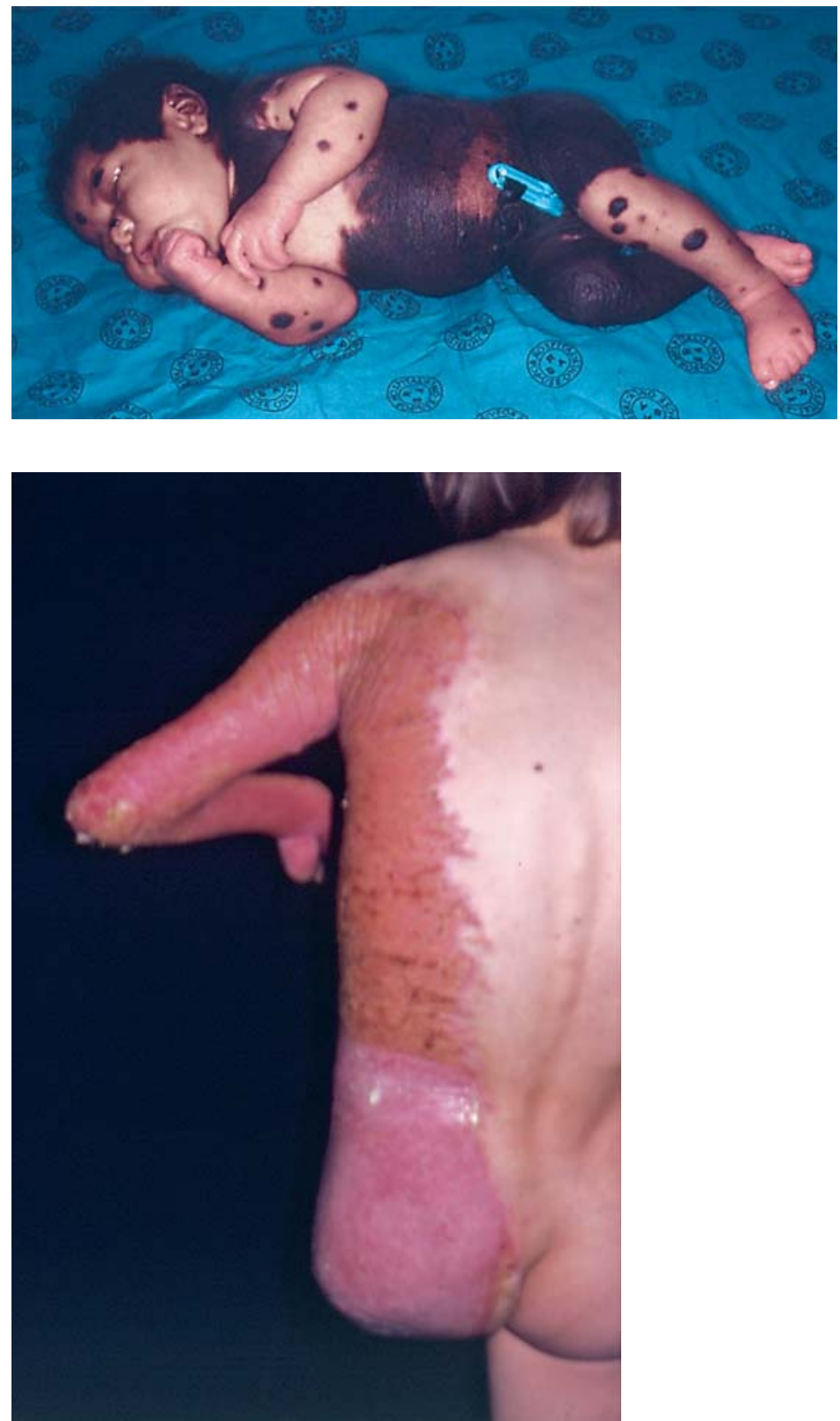

alisation in CHILD syndrome.

but it does not involve alteration of DNA sequence. Epigenetic causes are lyonisation, DNA methylation or demethylation, and retrotransposons.

Lyonisation means a random inactivation of one X-chromosome that will cause the genetic defect to be carried in some cells and be absent in others, thus leading to a mosaic pattern. Clinically, this genetic modification, rather than mutation, manifests in the lines of Blaschko. Retrotransposons are particles of retroviral origin that are interspersed in large amounts (40\%) throughout the human genome. They can affect the activity of adjacent genes by methylation or demethylation, resulting in silencing or activation of gene expression. Retrotransposons may give rise to phenotype variation in the form of variegated coat patterns reminiscent of the lines of Blaschko [26, 28].

The stage of development at which a genetic or epigenetic event occurred determines the extent of the phenotypic expression. If the mutation occurred early in embryogenesis, the 
abnormal clone will likely be widely distributed and affect multiple tissues, whereas a late mutation will be confined to distribution to the skin only [5].

An interesting point of view regarding the concept of skin mosaicism as a genetic basis for lmr has been brought to discussion by Rami Al-Rohil et al. [26]. They speculated that there are many pathologic processes (inflammatory, pigmentary, neoplasms) that, despite their divergent underlying causes, can follow the Blaschko linear pattern in their distribution, meaning that it must be a genetic predisposition that makes mosaic cells more susceptible to displaying other pathologies [26]. A similar opinion has been embraced by Findley, who stated that a pre-pattern, an "invisible blue-print", leads to linearity or partial expressivity of the lesions in areas of apparent symmetry [3]. Before the naevoid anomalies are generated, the affected segments of the skin must already exist at a cellular mosaic, capable of complex differentiation and rearrangement [3].

Reports of generalised cutaneous diseases sparing a mosaic area (LMR) are less common (alopecia universalis sparing a naevus flammeus, naevus depigmentosus spared by generalised drug reaction) $[5,26]$. The possibility exists that some reports labelled as reverse isotopic response, in the absence of a history of previous herpes zoster, may instead be cases of mosaic segments genetically resistant to generalised skin disorders such as drug reactions [26]. It is generally believed that the interplay between the susceptible cells that are present in a mosaic state and exposure to some unknown environmental factors triggers the eruption, rendering the mosaic abnormality clinically apparent $[5,26]$.

Perhaps a neglected aspect of clinical dermatology is the phenomenon of subjective irritation or sensitive skin, meaning those responses that have no clinically evident, objective expression, neither patterned nor generalised [29]. Does it mean that the disease is nonexistent? Kligman [29] made a few valuable observations regarding the occult changes of diseases. He believed that the visible lesions actually represent a late stage of disease and that histological skin changes can be demonstrated without having clinical expression. In acquired widespread skin diseases induced by exogenous factors or in patterned cutaneous lesions, "normal"-looking skin is always abnormal. It may quietly continue to register further damage for months or years as skin has a long-lasting memory. He stated that "venturing into subclinical universe is changing our traditional views of pathogenesis" [29].

\section{Conclusions}

The diversity of the skin patterns attempted to be described in this article points towards the richness of the original ideas and novel concepts, as well as to the value of descriptive dermatology in the era of evidence-based medicine. Identification of an ICD in dermatology is important for both diagnostic and prevention purposes, as a synchronous coexistence of skin disorders (autoimmune, malignancies) may be forecasted. Cutaneous mosaicism, proven in an increasing number of both congenital and acquired patterned skin disorders, plays an important role in locating the lesions. It explains the Blaschko linear patterns and the presence of congenitally determined vulnerable zones in the skin. Future genetic studies will help us piece together the puzzle of patterned distribution of skin diseases.

\section{Acknowledgement}

The clinical photographs used in this paper are from the archives of the Department of Dermatology, Steve Biko Academic Hospital, University of Pretoria. 


\section{Disclosure Statement}

There is no possible conflict of interest to preclude consideration of the paper for publication and no ethical bias.

\section{References}

Goldberg I, Sprecher E: Patterned disorders in dermatology. Clin Dermatol 2011;29:498-503.

Wollenberg A, Eames T: Skin diseases following a Christmas tree pattern. Clin Dermatol 2011;29:189-194.

Findley G: The genetic mosaic. J R Soc Med 1993;86:212-216.

4 Bolognia JL, Orlow SJ, Glick SA: Lines of Blaschko. J Am Acad Dermatol 1994;31:157-190.

-5 Molho-Pessach V, Schaffer JV: Blaschko lines and other patterns of cutaneous mosaicism. Clin Dermatol 2011; 29:205-225.

6 Rott HD: Extracutaneous analogies of Blaschko lines. Am J Med Genet 1999;85:338-341.

7 Lipsker D, Cribier B, Girard-Lemaire F: Genetic mosaicism in an acquired inflammatory dermatosis following the lines of Blaschko. Arch Dermatol 2000;136:805-807.

8 Happle R: Superimposed segmental manifestation of polygenic skin disorders. J Am Acad Dermatol 2007;57: 690-699.

-9 Lipsker D, Lenormand C: Classification of polygenic inflammatory diseases distributed along the lines of Blaschko. Dermatology 2009;219:99-101.

-10 Ruocco V, Ruocco E, Piccolo V, Brunetti G, Guerrera LP, Wolf R: The immunocompromised district in dermatology: a unifying pathogenic view of the regional immune dysregulation. Clin Dermatol 2014;32:569-576.

-11 Ruocco V, Ruocco E, Brunetti G, Sangiuliano S, Wolf R: Opportunistic localisation of skin lesions on vulnerable areas. Clin Dermatol 2011;29:483-488.

-12 Ruocco V, Brunetti RV, Ruocco E: The immunocompromised district: a unifying concept for lymphomatous, herpes-infected and otherwise damaged sites. J Eur Acad Dermatol Venereol 2009;23:1364-1373.

13 Wolf R, Brenner S, Ruocco V, Filioli FG: Isotopic response. Int J Dermatol 1995;34:341-348.

14 Ruocco V, Ruocco E, Ghersetich I, Bianchi B, Lotti T: Isotopic response after herpes virus infection: an update. J Am Acad Dermatol 2002;46:90-94.

-15 Ruocco V, Ruocco E, Brunetti G, Russo T, Gambardella A, Wolf R: Wolf's post-herpetic isotopic response: infections, tumours and immune disorders arising on the site of healed herpetic infections. Clin Dermatol 2014;32: 561-568.

16 Pakran J: Sparing phenomena in dermatology. Indian J Dermatol Venereol Leprol 2013;79:545-550.

-17 Wollenberg A, Burgdorf WH, Schaller M: Long-lasting "Christmas tree rash" in an adolescent: isotopic response of indeterminate cell histiocytosis in pityriasis rosea? Acta Derm Venereol 2002;82:288-291.

18 Huynh TN, Jackson JD, Brodell RT: Tattoo and vaccination sites: possible nest for opportunistic infections, tumours, and dysimmune reactions. Clin Dermatol 2014;32:678-684.

19 Wyburn-Mason R: Malignant change arising in tissues affected by herpes. Br Med J 1955;2:1106-1109.

20 Boyd AS, Neldner KH: The isomorphic response of Koebner. Int J Dermatol 1990;29:401-410.

21 Happle R, Van der Steen PHM, Perret C: The Renbok phenomenon: an inverse Koebner reaction observed in alopecia areata. Eur J Dermatol 1991;1:228-230.

-22 Tenea D: Carbamazepine-induced Stevens-Johnson syndrome sparing the skin previously affected by herpes zoster infection in a patient with systemic lupus erythematosus: a reverse isotopic phenomenon. Case Rep Dermatol 2010;2:140-145.

-23 Kroth J, Tischer J, Samtleben W, Weiss C, Ruzicka T, Wollenberg A: Isotopic response, Koebner phenomenon and Renbok phenomenon following herpes zoster. J Dermatol 2011;38:1058-1061.

24 Happle R: Dohi Memorial Lecture. New aspects of cutaneous mosaicism. J Dermatol 2002;29:681-692.

25 Findley GH, Moores PP: Pigment anomalies of the skin in the human chimaera: their relation to systematized naevi. Br J Dermatol 1980;103:489-498.

-26 Al-Rohil RN, Leung D, Carlson A: Congenital vulnerability of cutaneous segments arising from skin mosaicism: a genetic basis for locus minoris resistentiae. Clin Dermatol 2014;32:577-591.

-27 Happle R: The categories of cutaneous mosaicism: a proposed classification. Am J Med Genet A 2016;170A:452459.

28 Happle R: Transposable elements and the lines of Blaschko: a new perspective. Dermatology 2002;204:4-7.

29 Kligman AM: The invisible dermatoses. Arch Dermatol 1991;127:1375-1382. 